\title{
Research on heterogeneous compound flooding system based on dispersed particle gel
}

\author{
Hui $\mathrm{Li}^{1 \oplus *}$, Kai Wang ${ }^{2,3}$, Xiaodong $\mathrm{Han}^{1}$, Jinding Zheng ${ }^{1}$ \\ ${ }^{1}$ Tianjin Branch, China National Offshore Oil Corporation, Tianjin 300459, P. R. China \\ ${ }^{2}$ China National Offshore Oil Corporation Research Institute, Beijing 100028, P. R. China \\ ${ }^{3}$ State Key Laboratory of Offshore Oil Exploitation, Beijing 100028, P. R. China
}

(Received January 21, 2019; revised Feburary 19, 2019; accepted Feburary 20, 2019; available online Feburary 22, 2019)

Citation:

Li, H., Wang, K., Han, X., Zheng, J. Research on heterogeneous compound flooding system based on dispersed particle gel. Advances in Geo-Energy Research, 2019, 3(2): 156-164, doi: 10.26804/ager.2019.02.05.

Corresponding author:

*E-mail: lihui87@cnooc.com.cn

\section{Keywords:}

Dispersed particle gel

heterogeneous compound flooding system stability

performance evaluation

oil displacement mechanism

\begin{abstract}
:
Aiming at addressing the problem that the remaining oil maintains wide distribution after polymer flooding and the existing chemical compound flooding systems lack extensive application, a heterogeneous compound flooding system based on dispersed particle gel (DPG) is proposed as an alternative technology for enhanced recovery after polymer flooding. This study designs and characterizes the heterogeneous compound flooding system, as well as its impact factors, and field trial performances. With the systematic viscosity and oil-water interfacial tension as evaluation indicators, the field trial performance of the heterogeneous polymer flooding system is optimized and evaluated based on DPG. The results show that the systematic viscosity is 12.1 to $17.3 \mathrm{mPa} \cdot \mathrm{s}$ while the interfacial tension keeps constant at $10^{-3} \mathrm{mN} / \mathrm{m}$ magnitude. At $80{ }^{\circ} \mathrm{C}$, with 60 days aging, the viscosity retention ratio of the system is over $83.5 \%$, and the interfacial tension between oil and water is still maintained at $10^{-3} \mathrm{mN} / \mathrm{m}$. The results of core flooding experiment show that the heterogeneous compound flooding system exhibits good injection performance, moving characteristics of plugging, breakthrough migration and re-plugging. After hightemperature aging, subsequent water flooding still sustains at high pressure with high resistance to erosion. Through the selective high and low permeability layers, the water injection profile is improved, and eventually oil recovery is enhanced to $20.61 \%$ after polymer flooding.
\end{abstract}

\section{Introduction}

At present, the compound flooding becomes a new enhanced oil recovery technology after polymer flooding with its unique advantages which has a broad application prospect in oil fields (Carrero et al., 2007; Khan et al., 2009; Samanta et al., 2012; Olajire, 2014). Binary combination flooding system based on polymer and surfactant, ternary compound flooding system based on surfactant, polymer, alkaline, and heterogeneous compound flooding system based on preformed particle gel (PPG), polymer, are three main important technical means for recovering the remaining oil after polymer flooding. In the compound flooding system, the viscosity of the displacing phase is increased by the polymer, which leads to the improved flow ratio and the increased sweep volume. And the alkali and surfactant can make surfactant produce ultra low interfacial tension with oil in low concentration. Therefore, the compound flooding system can increase oil displacement efficiency to improve oil recovery (Nasr-El-Di et al., 1992; Nedjhioui et al., 2005; Samanta et al., 2011; Panthi and Mohanty, 2013).

However, in the field trial, the chemical compound flooding methods also expose some problems: During the polymer/ surfactant binary flooding process, the polymer is susceptible to the shear of mechanical equipment, formation pores and the physical and chemical properties of the formation, which causes a significant drop in the viscosity of the polymer and a decrease in fluid control. Alkali in ternary compound flooding system will cause polymer viscosity reduction, formation damage, wellbore fouling and crude oil emulsification and other negative effects. As to PPG heterogeneous flooding system, due to large particle size of PPG, the injection of PPG heterogeneous flooding system is poor, which leads to a limited range of formation permeability (Cash et al., 1977; Taber, 1981; Hou et al., 2005; Wever et al., 2013).

In order to further improve recovery after polymer flooding, a heterogeneous compound flooding system based on dispersed particle gel (DPG) is proposed in this paper to 


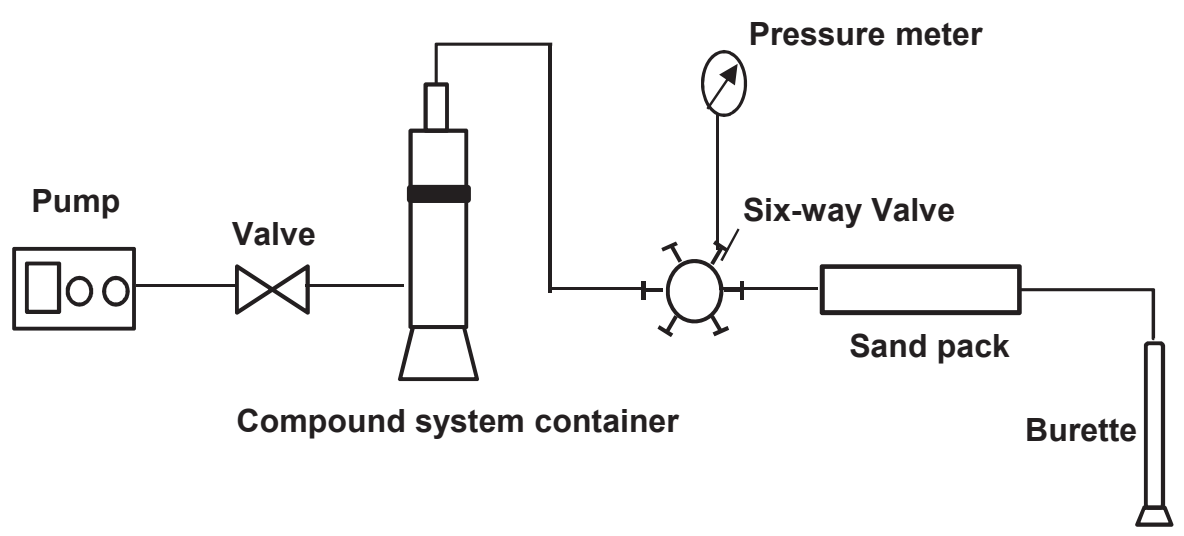

Fig. 1. The single-tube experimental diagram.

overcome the limitations of existing chemical flooding technologies. The system consists of low-concentration surfactants, polymers, and nano-sized DPG, and it improves oil recovery by synergistic action of polymer, surfactant and DPG.

\section{Experimental section}

\subsection{Materials}

The polymer (code: SDYF), relative molecular weight $9,800,000 \mathrm{~g} / \mathrm{mol}$, degree of hydrolysis $15.6 \%$. The surfactant (code: THSB) is tetradecyl sulfobetaine. The dispersed particle gels (DPG) is self-made in the laboratory. The crude oil is dehydrated and degassed oil from Changqing Oilfield. The brine is the simulated water of Changqing Oilfield. The salinity of the brine is about $37,000 \mathrm{mg} / \mathrm{L}$.

\subsection{Methods}

\subsubsection{Determination of particle size}

The Zetasizer Nano S dynamic light scattering instrument is used to measure the particle size of the heterogeneous compound flooding system. The experimental temperature is controlled at $80{ }^{\circ} \mathrm{C}$, the scattering angle is $173^{\circ}$, and the incident light wavelength is $633 \mathrm{~nm}$.

\subsubsection{Rheology measurement}

A rheometer (Model: Physica MCR 301; Anton Paar Ltd.) is used to measure the rheology of the heterogeneous compound flooding system. The relationship between the viscosity of oil displacement system and shear rate is measured under stable shear condition, and the experimental temperature is set at $80{ }^{\circ} \mathrm{C}$.

\subsubsection{Determination of microcosmic morphology}

The morphologies of the heterogeneous compound flooding system is observed by a transmission electron microscope. A drop of the sample solution is placed on a transmission electron microscopy (TEM) grid, and the excess solution is removed with filter paper. The TEM, operating at $80-100 \mathrm{kV}$, is used to observe the grid with the sample.

\subsubsection{Viscosity measurement}

The viscosity is measured by Brookfield DV-II Viscometer Rotor No.0 (6 r/min), and the experimental temperature is controlled at $80{ }^{\circ} \mathrm{C}$. Frist, $18-20 \mathrm{~mL}$ of the sample is injected into the sleeve at constant temperature for 15 minutes. Next, the viscometer is opened to record the viscosity value.

\subsubsection{Interfacial tension test}

The oil-water interfacial tension is measured using a Texas$500 \mathrm{C}$ interfacial tension meter according to the method described in references (Dai et al., 2015; Wang et al., 2016).

\subsubsection{Injection performance}

The injection capacity is determined using a single-tube physical simulation experiment. Fig. 1 demonstrates the experimental flow diagram. The experiment is operated using the following steps: (1) Fill the sand pack with brine, then calculate core pore volume and permeability; (2) Inject multiplier pore volume (PV) of heterogeneous compound flooding system into the sand pack until the pressure reaches stability, recording the pressure changes during the injection.

\subsubsection{Profiles improve performance test}

The profiles improving performance is determined by a double-tube physical model. The experimental flow chart is displayed in Fig. 2. The experiment is conducted using the following steps: (1) Fill high and low permeability sand pack separately; (2) Water flood to a stable displacement state, that is, the water production rate remains unchanged; (3) Inject polymer solution with one pore volume of the high permeability sand pack, and the injection concentration of polymer is $2,000 \mathrm{mg} / \mathrm{L}$. Put two sand packs into an oven at $80{ }^{\circ} \mathrm{C}$ for $24 \mathrm{~h}$; (4) Water flood to a stable displacement state again; (5) Inject heterogeneous compound flooding system with one pore volume of the high permeability sand pack, and the injection concentration of heterogeneous compound flooding system is $300 \mathrm{mg} / \mathrm{L}$ THSB $+1,000 \mathrm{mg} / \mathrm{L}$ SDYF + 


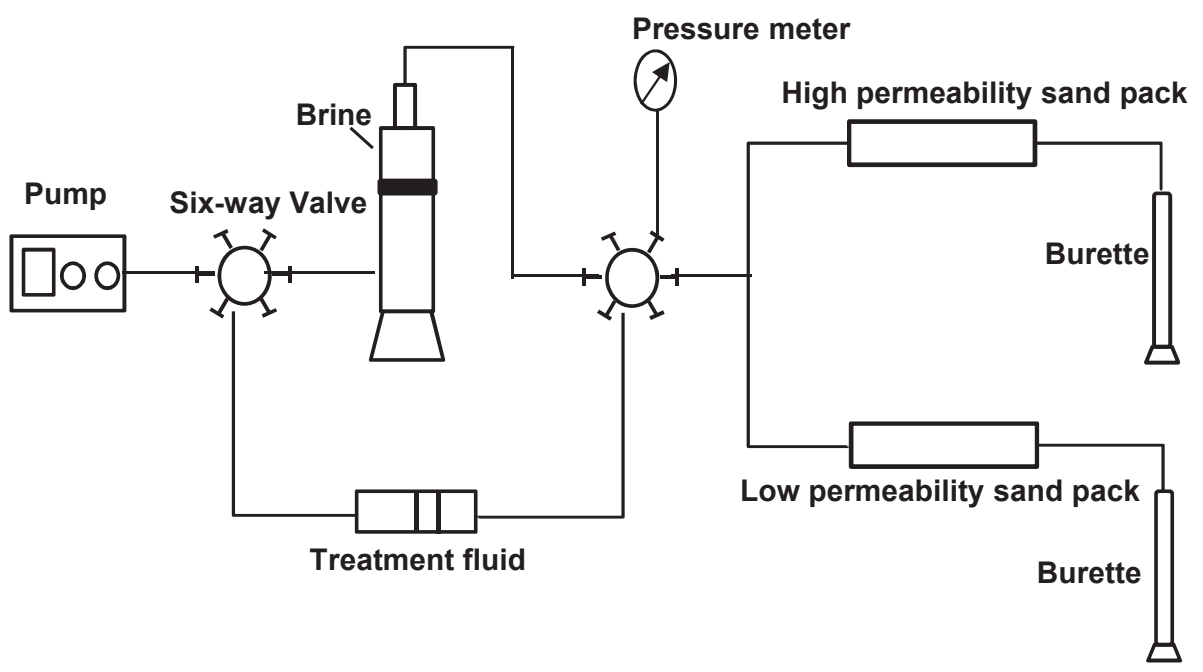

Fig. 2. The double-tube experimental diagram.

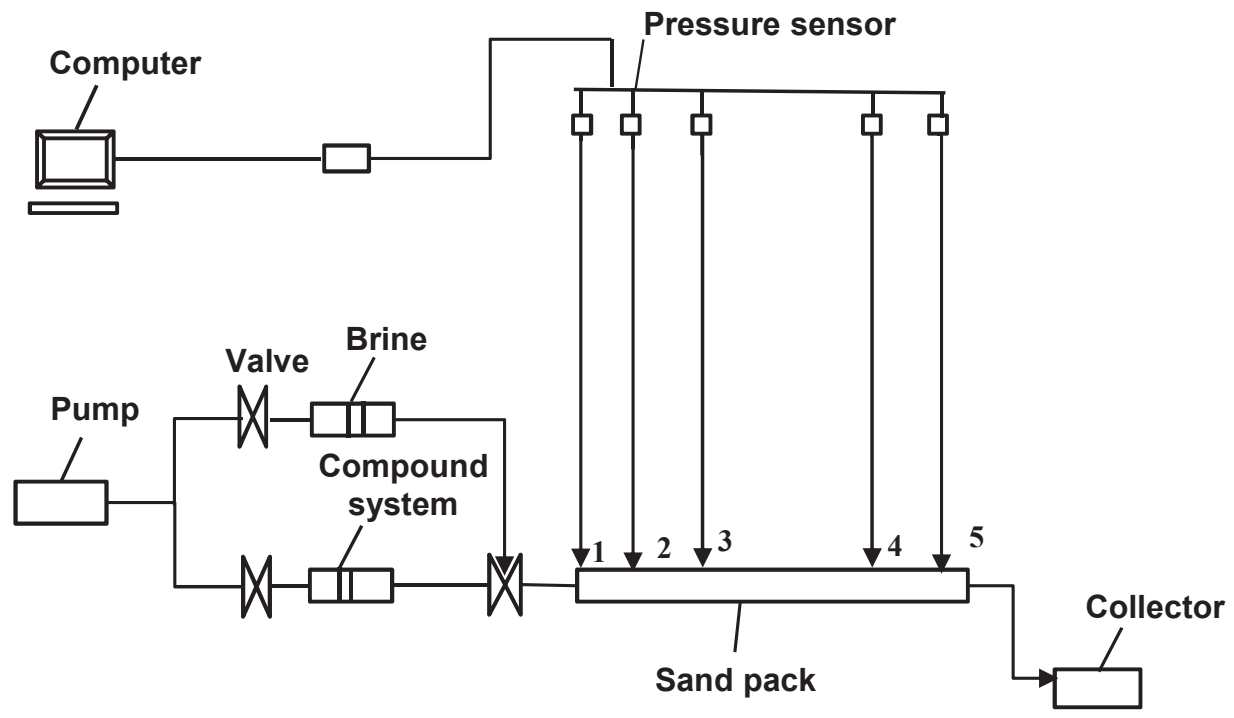

Fig. 3. The transport-blocking test experiment flow chart.

$1,000 \mathrm{mg} / \mathrm{L}$ DPG. Put two sand packs into an oven at $80{ }^{\circ} \mathrm{C}$ for 7 days; (6) Water flood to a stable displacement state again. The pressure changes and the fluid produced in the whole process are recorded. The water-produced rate of the high and low permeability sand pack is calculated according to the fluid produced.

\subsubsection{Determination of sealing-migration law}

The sealing-migration law of heterogeneous compound flooding system is investigated using the multi-point pressure measuring device (Zamani et al., 2010). The experimental flow chart is shown in Fig. 3. The steps for determining sealingmigration law are as follows: (1) Fill the sand pack with brine, then calculate core pore volume and permeability; (2) 0.3 pore volume (PV) of heterogeneous compound flooding system is injected into the sand pack; (3) Put the sand packs into an oven at $80{ }^{\circ} \mathrm{C}$ for 7 days; (4) Subsequent water flooding is performed until the pressure reaches a steady state, recording the pressure changes in the whole process.

\section{Results and discussion}

\subsection{Particle size of heterogeneous compound flood- ing system}

The Zetasizer Nano S Dynamic Light Scatterometer is used to measure the change of particle size before and after aging of the heterogeneous compound flooding system. Heterogeneous compound flooding system formula is a typical formula, and the concentration of which is $300 \mathrm{mg} / \mathrm{L} \mathrm{THSB}+1,000 \mathrm{mg} / \mathrm{L}$ $\mathrm{SDYF}+1,000 \mathrm{mg} / \mathrm{L} \mathrm{DPG}$. The aging time is 7 days. The 


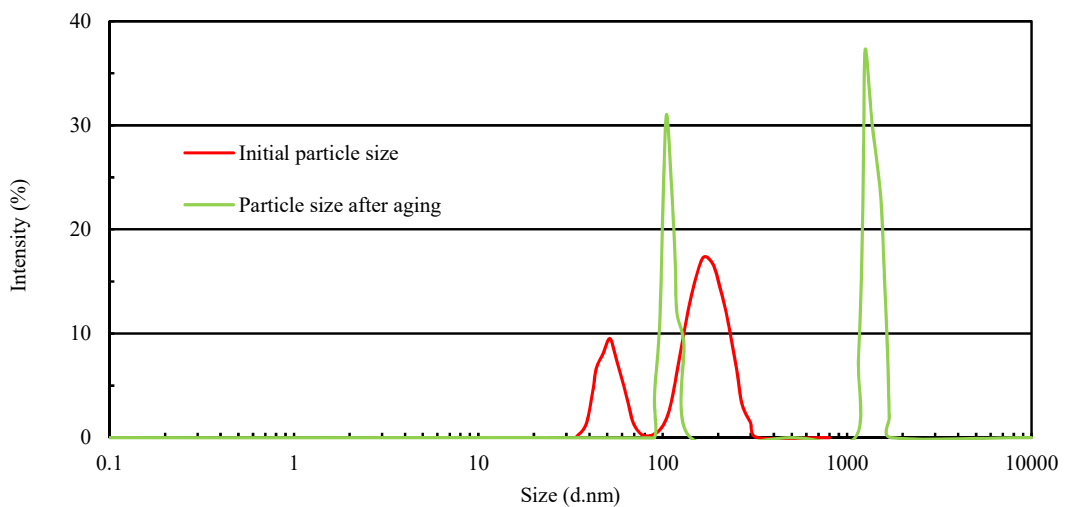

Fig. 4. Change in particle size of heterogeneous compound flooding system.

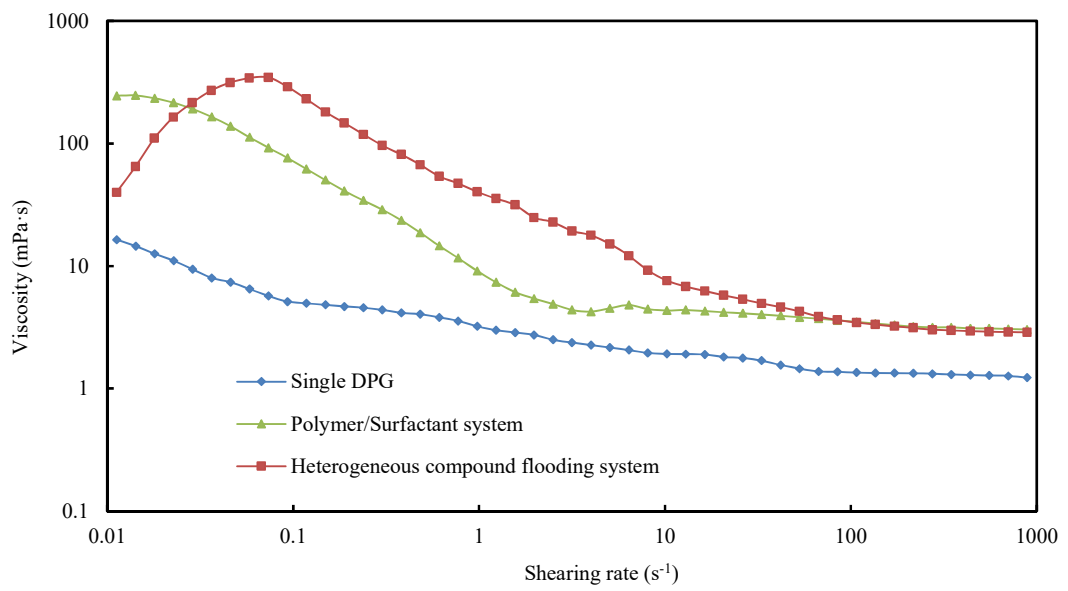

Fig. 5. System rheology.

experimental results are manifested in Fig. 4.

As depicted in Fig. 4, the system particle size increases with the increase of aging time, and the peak shifts to the right, the average particle size increases from $108 \mathrm{~nm}$ to $1,064.7 \mathrm{~nm}$. This is mainly due to the small particle size and large surface energy of the DPG, resulting in instability of the system. The aging process exacerbates the Brownian motion of the DPG, and the DPG agglomerate to form larger particles. The particle size of the system is increased. This aggregation behavior is favorable for flooding. When the system flows in the formation, the surfactants and polymer play an important role in increasing oil displacement efficiency and sweep volume; the DPG accumulate at the high-permeability channels or pore throat along with prolonged time, preventing the system breakthrough flow in the high-permeability channels, and the compound flooding system is diverted to medium and low permeability layer, increasing the sweep volume of the compound flooding system.

\subsection{Rheology of heterogeneous compound flooding system}

The rheology of a typical system is studied through Physica MCR 301 rheometer and compared with a single DPG system and a binary system based on polymer and surfactant. The experimental results are shown in Fig. 5.

From Fig. 5, it can be seen that the viscosity of the system increases with the increase of the shear rate at a low shear rate, which exhibits the rheological properties of the dilatant fluid, and it exhibits the rheological properties of the pseudoplastic fluid in the medium shear rate range, that is, the viscosity decreases with the increase of the shear rate. In the high shear rate range, the viscosity does not change with the increase of the shear rate, showing the rheological behavior of Newtonian fluid. Because the heterogeneous compound flooding system has the characteristics of polymer/surfactant binary flooding system, and the addition of DPG leads to the unique rheological properties of the system. At low shear rate, shear stress is not enough to dismantle the physical association structure between polymer molecules and the aggregation state of DPG in polymer solution. The free movement of DPG becomes difficult, and the interaction force between particles increases. As a result, the flow resistance increases, and the viscosity of the system increases with the increase of shear rate, showing the rheological properties of the dilatant fluid. The physical association structure between polymer molecules and the aggregation state of DPG in polymer solution are gradually destroyed in the medium shear rate range, and the 


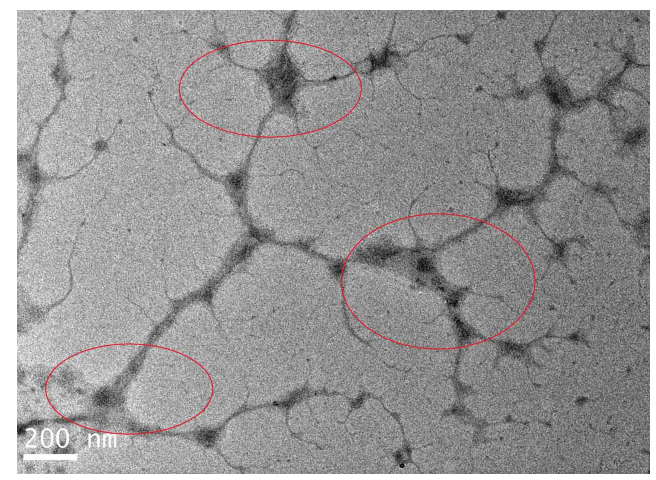

(a)

Fig. 6. Micromorphology before aging.

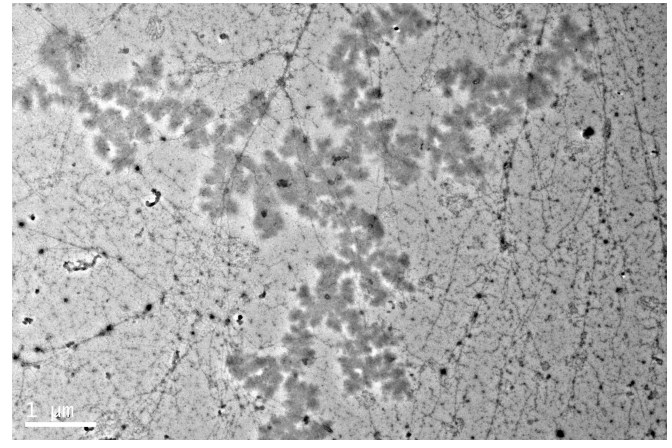

(a)

Fig. 7. Micromorphology after aging.

viscosity of the system decreases with the increase of shear rate, showing the rheological properties of pseudoplastic fluid. At high shear rate, the polymer molecular structure and the aggregation state of DPG are destroyed under the influence of shear stress, and the viscosity of the system has little change with the increase of shear rate, showing the rheological behavior of Newtonian fluid.

\subsection{Microcosmic morphology of heterogeneous compound flooding system}

Figs. 6 and 7 display the microstructure of the typical system before and after aging using TEM. It can be seen that the DPG mainly exist in the form of a single particle before the aging of the heterogeneous compound flooding system, the particles are not continuous, the aggregation behavior is not obvious, and the particle size of the particles is around 100 $\mathrm{nm}$. After aging for 7 days, the DPG aggregate with each other to form continuous and larger aggregates with particle size up to $10 \mu \mathrm{m}$. Moreover, the polymer SDYF and the surfactant THSB form a continuous film by adsorbing on the surface of DPG, which facilitates the mutual aggregation of the particles to form larger aggregates and maintains this stable aggregation morphology. This is conducive to giving full play to the deep profile control and flooding performance of the DPG.

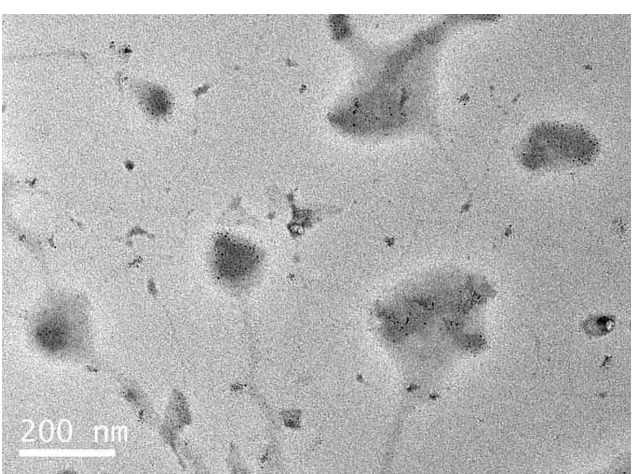

(b)

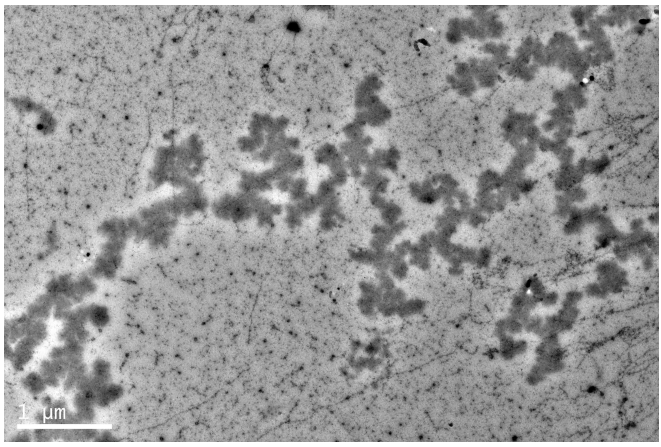

(b)

\subsection{Viscosity of heterogeneous compound flooding system}

\subsubsection{Temperature resistance}

The temperature resistance of the viscosity of the heterogeneous compound flooding system is investigated (Fig. 8), and Formula 1 is $300 \mathrm{mg} / \mathrm{L}$ THSB $+1,000 \mathrm{mg} / \mathrm{L} \mathrm{SDYF} \mathrm{+}$ $3,000 \mathrm{mg} / \mathrm{L}$ DPG, Formula 2 is $300 \mathrm{mg} / \mathrm{L}$ THSB $+1,500 \mathrm{mg} / \mathrm{L}$ $\mathrm{SDYF}+3,000 \mathrm{mg} / \mathrm{L}$ DPG, Formula 3 is $300 \mathrm{mg} / \mathrm{L} \mathrm{THSB}+$ $2,000 \mathrm{mg} / \mathrm{L} \mathrm{SDYF}+3,000 \mathrm{mg} / \mathrm{L} \mathrm{DPG}$, Formula 4 is $600 \mathrm{mg} / \mathrm{L}$ THSB + 1,000 mg/L SDYF + 3,000 mg/L DPG, Formula 5 is $600 \mathrm{mg} / \mathrm{L} \mathrm{THSB}+1,500 \mathrm{mg} / \mathrm{L} \mathrm{SDYF}+3,000 \mathrm{mg} / \mathrm{L} \mathrm{DPG}$, Formula 6 is $600 \mathrm{mg} / \mathrm{L}$ THSB + 2,000 mg/L SDYF + 3,000 $\mathrm{mg} / \mathrm{L}$ DPG.

From Fig. 8, the viscosity of the heterogeneous compound flooding system decreases with the increase of temperature, the viscosity is still greater than $12 \mathrm{mPa} \cdot \mathrm{s}$ at $90{ }^{\circ} \mathrm{C}$, showing good temperature resistance performance.

\subsubsection{Salt resistance}

The heterogeneous compound flooding system is prepared by brine with different $\mathrm{NaCl}$ concentration, and the variation of the system viscosity with $\mathrm{NaCl}$ concentration is investigated (Fig. 9).

Fig. 9 shows that with the increase of $\mathrm{NaCl}$ concentration, the viscosity of the heterogeneous compound flooding system 


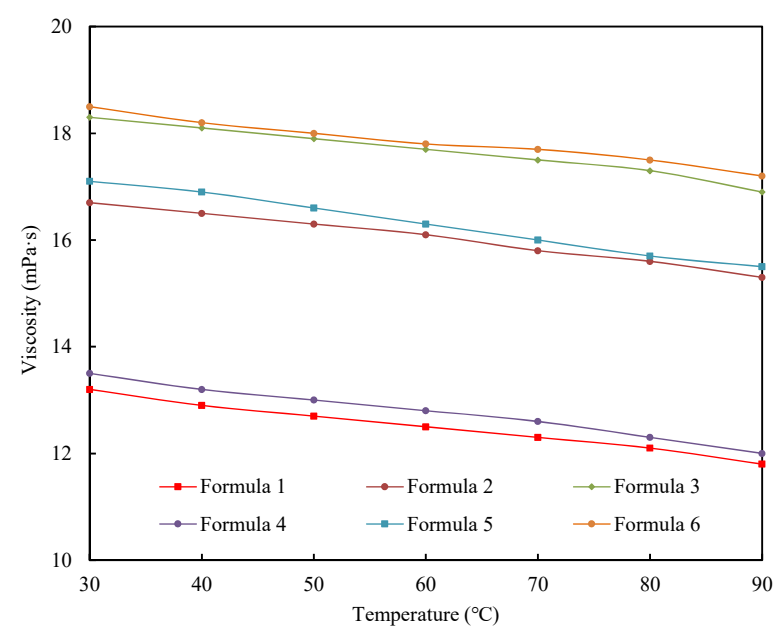

Fig. 8. Influence of temperature on viscosity of heterogeneous compound flooding system.

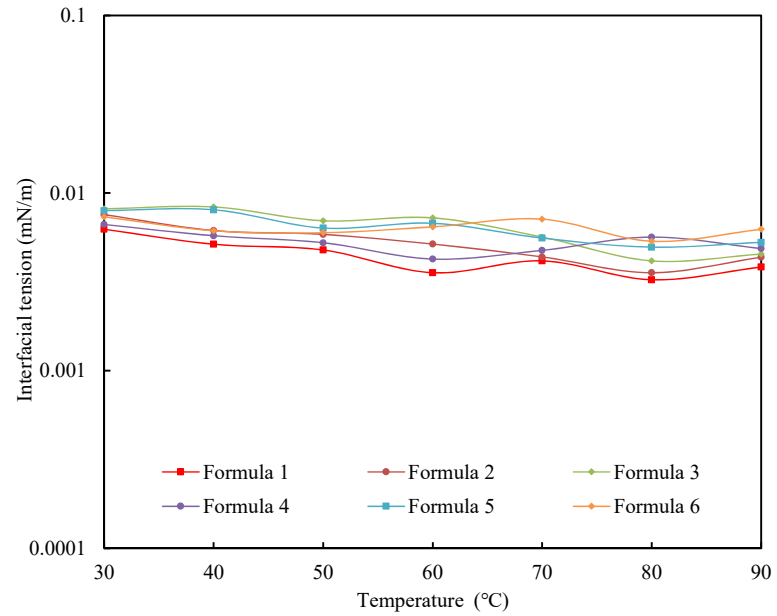

Fig. 10. Effect of temperature on interfacial tension of heterogeneous compound flooding system.

is significantly reduced, and the main reason is that the increase of $\mathrm{NaCl}$ concentration results in curling of polymer molecules, and the polymer can not be fully stretched and the viscosity of the system decreases.

\subsection{Interfacial tension of heterogeneous compound flooding system}

\subsubsection{Effect of temperature}

At different temperatures, the temperature resistance of the typical system to reduce the oil-water interfacial tension is investigated (Fig. 10), and Formula 1 is $300 \mathrm{mg} / \mathrm{L}$ THSB + $1,000 \mathrm{mg} / \mathrm{L}$ SDYF $+3,000 \mathrm{mg} / \mathrm{L}$ DPG, Formula 2 is $300 \mathrm{mg} / \mathrm{L}$ THSB + 1,500 mg/L SDYF + 3,000 mg/L DPG, Formula 3 is $300 \mathrm{mg} / \mathrm{L}$ THSB $+2,000 \mathrm{mg} / \mathrm{L} \mathrm{SDYF}+3,000 \mathrm{mg} / \mathrm{L} \mathrm{DPG}$, Formula 4 is $600 \mathrm{mg} / \mathrm{L}$ THSB $+1,000 \mathrm{mg} / \mathrm{L}$ SDYF $+3,000$ $\mathrm{mg} / \mathrm{L}$ DPG, Formula 5 is $600 \mathrm{mg} / \mathrm{L}$ THSB $+1,500 \mathrm{mg} / \mathrm{L}$ SDYF $+3,000 \mathrm{mg} / \mathrm{L}$ DPG, Formula 6 is $600 \mathrm{mg} / \mathrm{L}$ THSB $+2,000$ $\mathrm{mg} / \mathrm{L} \mathrm{SDYF}+3,000 \mathrm{mg} / \mathrm{L}$ DPG.

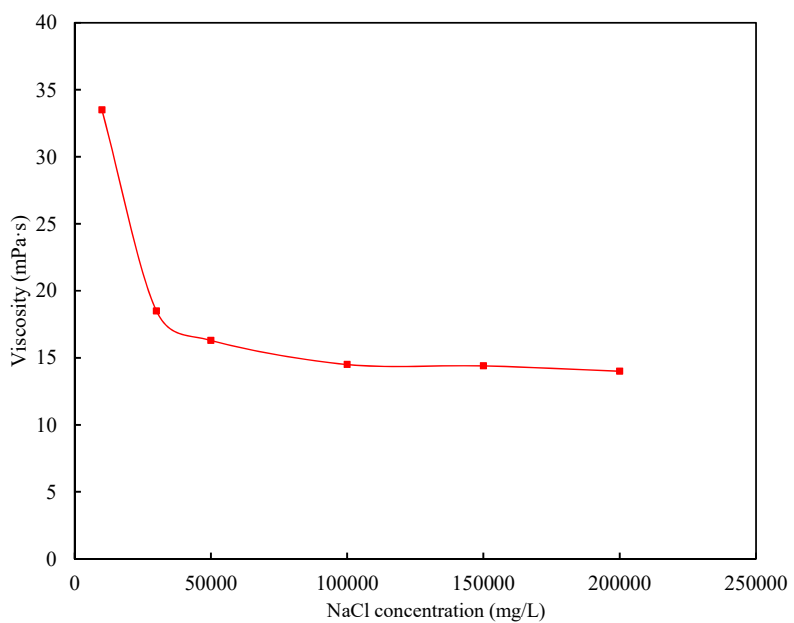

Fig. 9. Viscosity of different $\mathrm{NaCl}$ concentrations.

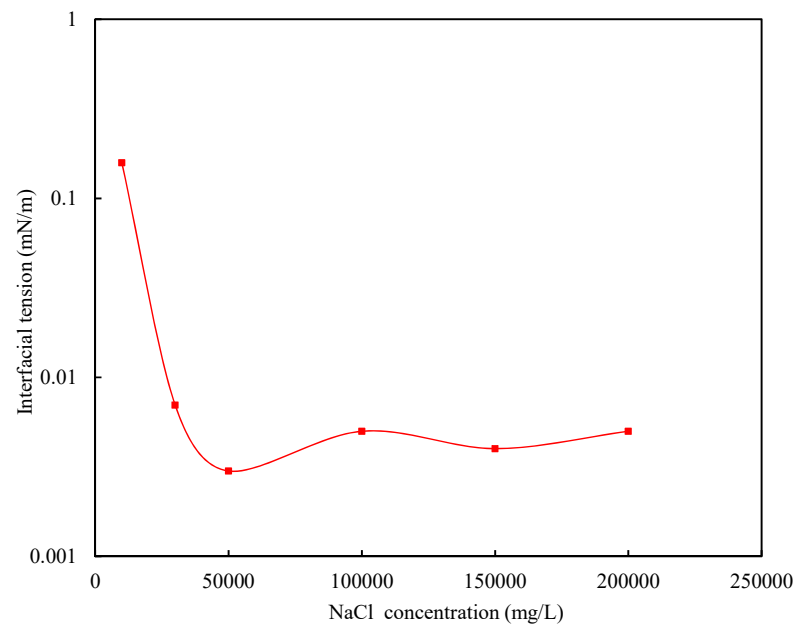

Fig. 11. Interfacial tension of different $\mathrm{NaCl}$ concentrations.

It is illustrated in Fig. 10 that the interfacial tension of the system is basically unchanged. The interfacial tension between the oil and the formation water remains at the order of $10^{-3} \mathrm{mN} / \mathrm{m}$, which shows good temperature resistance performance.

\subsubsection{The influence of salinity}

A typical system is prepared with different concentrations of $\mathrm{NaCl}$ concentration, and the oil-water interfacial tension between the system and the oil is investigated with different $\mathrm{NaCl}$ concentration (Fig. 11).

Fig. 11 indicates that with the increase of $\mathrm{NaCl}$ concentration, the oil-water interfacial tension between the heterogeneous compound flooding system and the oil decreases significantly at first and then stabilizes, showing good salt tolerance. This is mainly due to the fact that the addition of $\mathrm{NaCl}$ within a proper range adjusts the polarity of the oil phase and the aqueous phase, the lipophilic and hydrophilic properties of the surfactant are fully balanced, thereby maximizing the adsorp- 


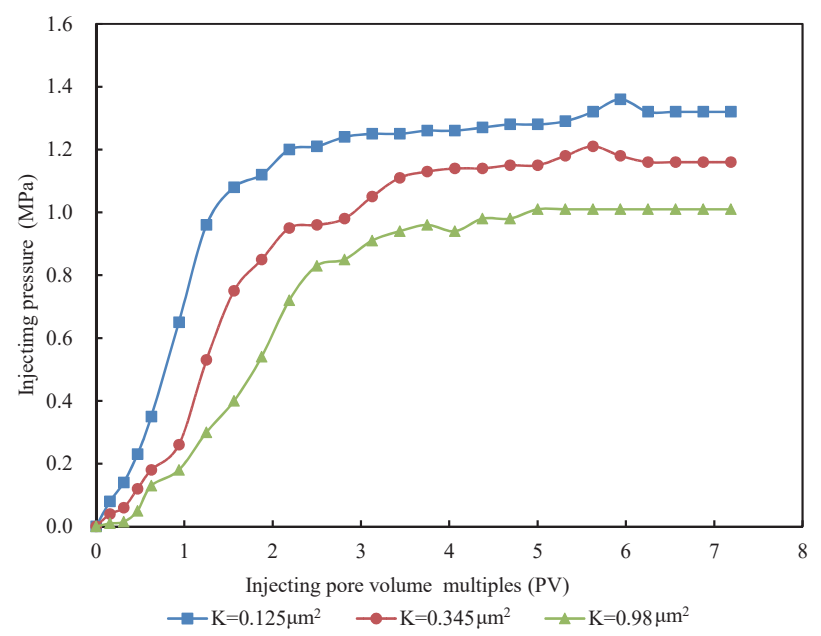

Fig. 12. Injectivity of heterogeneous compound flooding system with different permeability.

tion at the oil-water interface. It produces a lower interfacial tension (Babu et al., 2015).

\subsection{Injection performance}

Cores with different permeability are used to investigate the injection performance of the heterogeneous compound flooding system. The injection performance of the heterogeneous compound flooding system is demonstrated in Fig. 12.

As depicted in Fig. 12, under different permeability, the injection capacity of the system is different. For a typical heterogeneous compound flooding system formulation, the higher the permeability, the lower the steady injection pressure and the better the injection property.

\subsection{Profiles improve performance}

The double-tube experimental model is used to determine the selective plugging performance of the system (Zhao et al., 2014). The permeability ratio of the double-tube model is 3 , of which the permeability of high-permeability tube is $0.96 \mu \mathrm{m}^{2}$ and the permeability of low-permeability tube is $0.32 \mu \mathrm{m}^{2}$.

As depicted in Fig. 13, the system can effectively block the high permeability layer and adjust the seepage profile. This is due to the fact that the system preferentially enters larger pores under the injection pressure, and selectively blocks the high permeability layer through the bridging and adsorption of the DPG in the compound system, forcing the subsequent water flood to the low permeability layer. Then it adjusts the seepage profile of the high and low permeability layers and effectively improve the heterogeneity of the formation.

\subsection{Oil recovery determination}

The double-tube experimental model is used to determine the recovery of the oil displacement system (Liu et al., 2016). The experiment stages are as follows: (1) Saturate the core with brine; (2) Saturate crude oil in sand pack cores; (3) Water

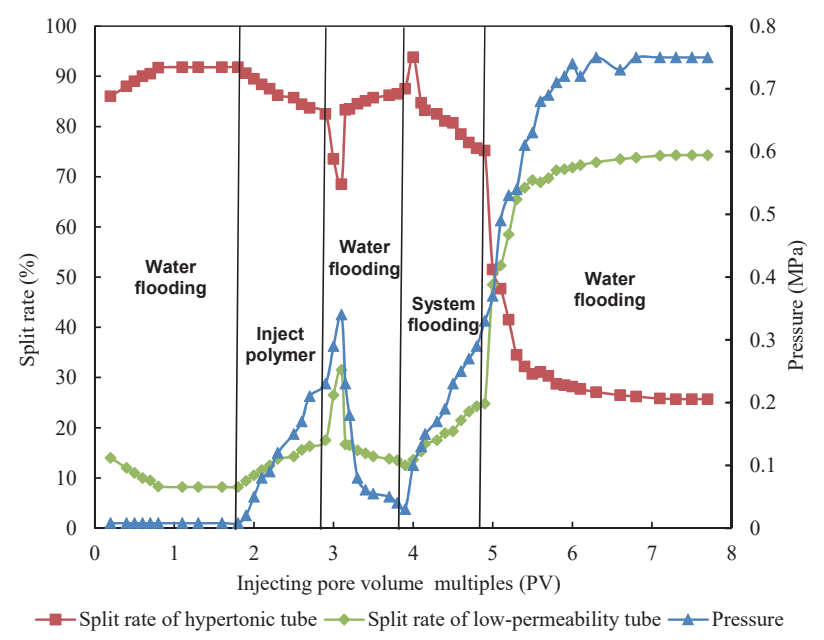

Fig. 13. Selective plugging capability of composite system.

flood until effluent water cut is around $98 \%$ for the crude oil saturated sand pack cores; (4) Inject $0.3 \mathrm{PV}$ of polymer solution. Put two sand packs into an oven at $80{ }^{\circ} \mathrm{C}$ for $24 \mathrm{~h}$; (5) Subsequent water flooding is applied until effluent water cut is around 98\% again. Calculate oil recovery increment of polymer flooding; (6) Inject $0.3 \mathrm{PV}$ of heterogeneous compound flooding system using the typical formula (300 $\mathrm{mg} / \mathrm{L}$ THSB $+1,000 \mathrm{mg} / \mathrm{L}$ SDYF $+1,000 \mathrm{mg} / \mathrm{L} \mathrm{DPG})$. Put two sand packs into an oven at $80{ }^{\circ} \mathrm{C}$ for 7 days; (7) Subsequent water flooding is applied until effluent water cut is around 98\% again. Calculate oil recovery increment of the heterogeneous compound flooding system. In the experiment, the high permeability sand pack (permeability: $0.94 \mu \mathrm{m}^{2}$ ) and the low permeability sand pack (permeability: $0.28 \mu \mathrm{m}^{2}$ ) are used to simulate a heterogeneous formation. The injection concentration of polymer is $2,000 \mathrm{mg} / \mathrm{L}$. The results of the double-pipe sand-fill model are manifested in Fig. 14.

It can be seen from Fig. 14 that the oil recovery increment of the heterogeneous compound flooding system is $9.12 \%$ higher than that of polymer/surfactant binary combination flooding system after polymer flooding. It is due to the limited adjustment ability of seepage profile of the poly/surfactant binary combination flooding system, and the heterogeneous compound flooding system not only has the function of increasing viscosity of polymer and reducing interfacial tension between oil and water by surfactant, but also has the effect of the deep profile control and displacement by DPG to improve oil recovery. In the heterogeneous compound flooding system, the aging DPG can form aggregates to plug high permeability channels through adsorption and bridging action, and effectively adjust seepage profile to improve the development effect of low permeability layer, and the increment of recovery is $20.61 \%$.

\subsection{Sealing-migration law in porous media}

A porous pressure measuring device (length $1 \mathrm{~m}$, diameter $2.5 \mathrm{~cm}$ ) is used to determine the migration of the typical systems in porous media. The pore volume of the sand-filled 


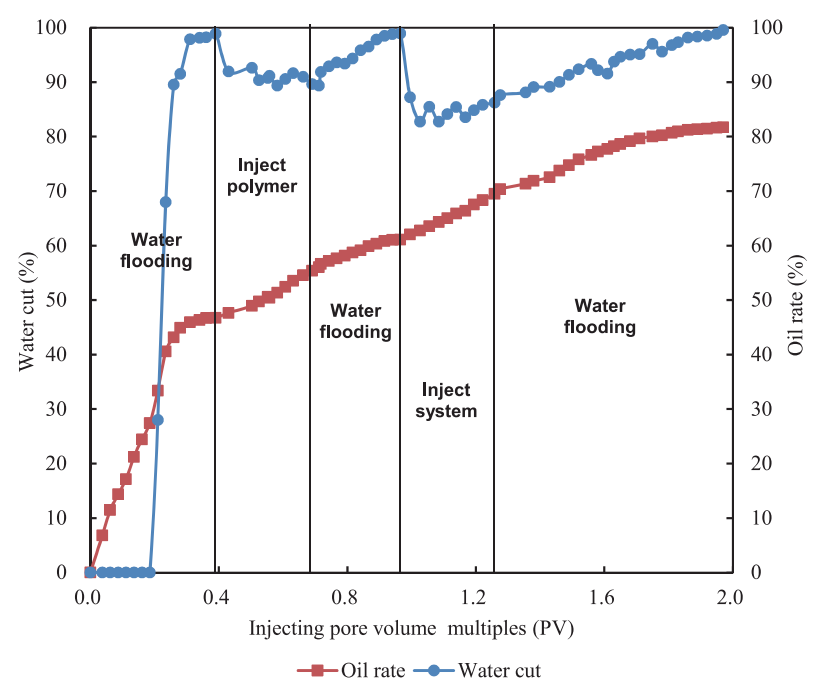

Fig. 14. Displacement effect of heterogeneous compound flooding system.

pipe is $128 \mathrm{~mL}$, and the water permeability is $188.6 \times$ $10^{-3} \mu \mathrm{m}^{2}$. The displacement results are illustrated in Fig. 15 .

As depicted in Fig. 15, the movement characteristics of plugging, breakthrough migration, and re-blocking are shown in the system injection and subsequent water flooding. In the process of injection of heterogeneous compound flooding system, the pressure of the pressure measurement points 1 and 2 increases rapidly with the increase of the injection volume, and the pressure difference between the pressure measurement points 1 and 2 is small, indicating that the system migrates well between the two pressure measurement points. And the pressure measuring points 1 and 2 are $100 \mathrm{~mm}$ and $300 \mathrm{~mm}$ away from the injection end, respectively. However, the pressures of the two pressure measuring points are very high, which indicates that the system generates additional resistance when it passes through the pore throat; the pressure difference between the pressure measuring points 4 and 5 during the subsequent water flooding is small, indicating that the system moves smoothly between the two pressure measuring points. The large pressure difference between the pressure measurement point 3 and 4 indicates that after the system migrates through the pressure measuring point 3 , a high strength plugging is formed due to the increase of the retention and the physical plugging of the pore throat by DPG. The pressure fluctuates continuously in a small range during subsequent water flooding, which reflects the continuous migration, blocking, remobilization and redistribution of the heterogeneous composite flooding system. And the injection pressure remains high throughout the process, indicating that the system has good resistance to erosion (Dai et al., 2017; Gong et al., 2017).

\section{Conclusion}

(1) Heterogeneous compound flooding system with DPG after aging for 60 days at a target reservoir temperature of $80^{\circ} \mathrm{C}$, the viscosity retention ratio of the system is more than $83.5 \%$, and the interfacial tension with the oil in the formation

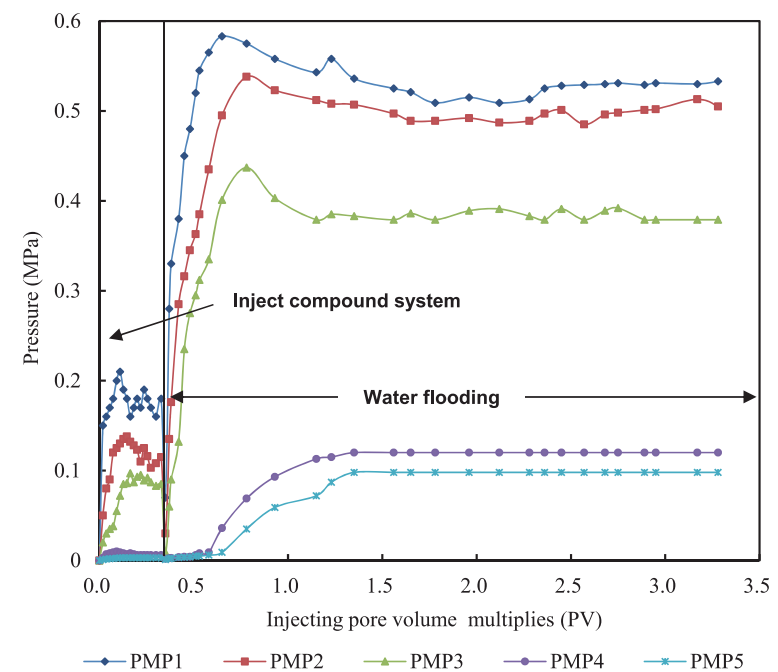

Fig. 15. Sealing and transport characteristics of heterogeneous compound flooding system.

maintains at the order of $10^{-3} \mathrm{mN} / \mathrm{m}$. The addition of salt contributes to the ability of the system to reduce the oil-water interfacial tension.

(2) In the heterogeneous compound flooding system, the DPG with each other to form larger particle size aggregates with the prolongation of the aging time. The addition of the surfactant and the polymer is performed on the surface of the DPG. The formation of a continuous film exacerbates the aggregation of the DPG, which is helpful to exert the deep profile control and displacement function of DPG and expand the sweep coefficient of the heterogeneous compound flooding system.

(3) At the low shear rate, the heterogeneous compound system exhibits the rheological properties of the dilatant fluid. In the medium shear rate range, the system exhibits the rheological characteristics of the pseudoplastic fluid. In the high shear rate range, the system exhibits rheological characteristics of Newtonian fluids.

(4) The core flow experiment shows that: The heterogeneous compound flooding system with DPG has good injection performance. It has the moving characteristics of plugging, breakthrough migration and re-blocking through pore throat after polymer flooding. It maintains high pressure after subsequent water flooding of multiple pore volumes, showing good resistance to erosion. It improves the water injection profile through selective sealing of high and low permeability layers. It enhances oil recovery about $20.61 \%$ after polymer flooding.

\section{Acknowledgments}

This research was conducted under the support of the National Science and Technology Major Project of China (Grant No. 2016ZX05058-003). The authors express their appreciation to technical reviewers for their constructive comments.

Open Access This article is distributed under the terms and conditions of the Creative Commons Attribution (CC BY-NC-ND) license, which permits 
unrestricted use, distribution, and reproduction in any medium, provided the original work is properly cited.

\section{References}

Babu, K., Pal, N., Bera, A., et al. Studies on interfacial tension and contact angle of synthesized surfactant and polymeric from castor oil for enhanced oil recovery. Appl. Surf. Sci. 2015, 353: 1126-1136.

Carrero, E., Queipo, N.V., Pintos, S., et al. Global sensitivity analysis of Alkali-Surfactant-Polymer enhanced oil recovery processes. J. Pet. Sci. Eng. 2007, 58(1-2): 30-42.

Cash, L., Cayias, J.L., Fournier, G., et al. The application of low interfacial tension scaling rules to binary hydrocarbon mixtures. J. Colloid Interface Sci. 1977, 59(1): 39-44.

Dai, C., Liu, Y., Zou, C., et al. Investigation on matching relationship between dispersed particle gel (DPG) and reservoir pore-throats for in-depth profile control. Fuel 2017, 207: 109-120.

Dai, C., Wang, K., Liu, Y., et al. Reutilization of fracturing flow back fluids in surfactant flooding for enhanced oil recovery. Energy Fuels 2015, 29(4): 2304-2311.

Gong, H., Zhang, H., Xu, L., et al. Further enhanced oil recovery by branched-preformed particle gel/HPAM/surfactant mixed solutions after polymer flooding in parallelsandpack models. RSC Adv. 2017, 7(63): 39564-39575.

Hou, J., Liu, Z., Zhang, S., et al. The role of viscoelasticity of alkali/surfactant/polymer solutions in enhanced oil recovery. J. Pet. Sci. Eng. 2005, 47(3-4): 219-235.

Khan, M.Y., Samanta, A., Ojha, K., et al. Design of Alkaline/Surfactant/Polymer (ASP) slug and its use in enhanced oil recovery. Pet. Sci. Technol. 2009, 27(17): 1926-1942.

Liu, Y., Dai, C., Kai, W., et al. Investigation on preparation and profile control mechanisms of the dispersed particle gels (DPG) formed from phenol-formaldehyde crosslinked polymer gel. Ind. Eng. Chem. Res. 2016, 55(22): 6284-6292.

Nasr-El-Di, H.A., Hawkins, B.F., Green, K.A. Recovery of residual oil using the alkali/surfactant/polymer process:
Effect of alkali concentration. J. Pet. Sci. Eng. 1992, 6(4): 381-401.

Nedjhioui, M., Moulai-Mostefa, N., Morsli, A., et al. Combined effects of polymer/surfactant/oil/alkali on physical chemical properties. Desalination 2005, 185(13): 543-550.

Olajire, A.A. Review of ASP EOR (alkaline surfactant polymer enhanced oil recovery) technology in the petroleum industry: Prospects and challenges. Energy 2014, 77: 963-982.

Panthi, K., Mohanty, K.K. Effect of alkaline preflush in an alkaline-surfactant-polymer flood. Energy Fuels 2013, 27(2): 764-771.

Samanta, A., Bera, A., Ojha, K., et al. Comparative studies on enhanced oil recovery by alkali-surfactant and polymer flooding. J. Pet. Explor. Prod. Technol. 2012, 2(2): 67-74.

Samanta, A., Ojha, K., Mandal, A. Interactions between acidic crude oil and alkali and their effects on enhanced oil recovery. Energy Fuels 2011, 24(4): 1642-1649.

Taber, J.J. Research on enhanced oil recovery: Past, present and future, in Surface Phenomena in Enhanced Oil Recovery, edited by D.O. Shah, Plenum Press, New York, pp. 13-52, 1981.

Wang, K., Liu, C., Zhou, W. Investigation on interfacial properties of viscoelastic-based surfactant as an oil displacement agent recovered from fracturing flow back fluid. RSC Adv. 2016, 6(44): 38437-38446.

Wever, D.A.Z., Picchioni, F., Broekhuis, A.A. Comblike polyacrylamides as flooding agent in enhanced oil recovery. Ind. Eng. Chem. Res. 2013, 52(46): 1635216363.

Zamani, A., Maini, B., Pereira-Almao, P. Experimental study on transport of ultra-dispersed catalyst particles in porous media. Energy Fuels 2010, 24(9): 4980-4988.

Zhao, G., Dai, C., Zhao, M. Investigation of the profile control mechanisms of dispersed particle gel. PLOS ONE 2014, 9(6): e100471. 